\title{
Randomized Double-Blind Clinical Trial of Eutectic Mixture of Local Anesthetic Creams in Reducing Pain During Hysterosalpingography
}

\author{
Mojgan Kalantari ${ }^{1}$; Shahrzad Zadeh Modares ${ }^{2}$; Firoozeh Ahmadi $^{3,{ }^{*}}$; Vajihe Hazari ${ }^{4}$; Hadieh \\ Haghighi $^{3}$; Mohammad Chehrazi ${ }^{5}$; Melika Razaghi ${ }^{6}$ \\ ${ }^{1}$ Department of Radiology, Mahdieh Hospital, Infertility and Reproductive Health Research Center (IRHRC), Shahid Beheshti University of Medical Sciences, Tehran, Iran \\ 2 Infertility and Reproductive Health Research Center (IRHRC), Shahid Beheshti University of Medical Sciences, Tehran, Iran \\ ${ }_{3}^{3}$ Department of Reproductive Imaging at Reproductive Biomedicine Research Center, Royan Institute for Reproductive Biomedicine, ACECR, Tehran, Iran \\ ${ }_{5}^{4}$ Mahdieh Hospital, Shahid Beheshti University of Medical Sciences, Tehran, Iran \\ ${ }_{6}$ Mepartment of Epidemiology and Reproductive Health at Reproductive Epidemiology Research Center, Royan Institute for Reproductive Biomedicine, ACECR, Tehran, Iran \\ *Corresponding author: Firoozeh Ahmadi, Department of Reproductive Imaging at Reproductive Biomedicine Research Center, Royan Institute for Reproductive Biomedicine, \\ ACECR, Tehran, Iran. Tel: +98-9121503522, E-mail: dr.ahmadi1390@gmail.com
}

\section{Received: January 31, 2013; Revised: June 16, 2013; Accepted: August 7, 2013}

Background: Hysterosalpingography (HSG) is considered as a primary test in infertility work up worldwide due to its reliability in evaluating abnormalities related to the uterus and fallopian tubes.

Objectives: To assess the efficacy of applying eutectic mixture of local anesthetics (lidocaine-prilocaine cream) (EMLA) on the uterine cervix in reducing pain during HSG.

Patients and Methods: Eighty patients undergoing HSG as part of infertility evaluation were randomly allocated to groups receiving either EMLA $(\mathrm{N}=40)$ or placebo cream $(\mathrm{N}=40)$ in a double-blinded prospective study. Fifteen minutes before HSG, 5 grams of $5 \% \mathrm{cream}$ was applied to the uterine cervix using a cervical applicator. The degree of pain experienced by the patient was evaluated during and after HSG at five predefined steps on a visual analogue scale(VAS).

Results: There was no significant difference in the efficacy between EMLA and placebo creams in pain perception during the entire procedure. There was no significant difference in long term pain perception half an hour after the HSG performance.

Conclusions: This study does not support the use of EMLA for HSG.

Keywords: Hysterosalpingography; Eutectic Lidocaine-Prilocaine; Pain; Visual Analog Scale

\section{Background}

Hysterosalpingography (HSG) is considered as a primary test in infertility work up worldwide due to its reliability in evaluating abnormalities related to the uterus and fallopian tubes. Despite rare complications, lower abdominal pain is found as the patient's main complaint during and after test performance (1). A number of techniques have been introduced to reduce the severity of pain, including substituting balloon catheter instead of a metal canola or using water based contrast media (25). Pain medications before and during HSG such as oral non-steroidal anti-inflammatory drugs or intravenous opioid analgesics have also been used $(6,7)$. Topical pain medication and local anesthetics either inserted into the uterus or applied on the uterine cervix before the procedure have been also examined in different studies $(8,9)$. Liberty et al. reported that eutectic mixture of local anesthetics (lidocaine-prilocaine) (EMLA) applied on the cervix significantly decreased discomfort during cervical instrumentation, which is found as the most painful step, and no complication was declared (1). With increased demand for HSG, mainly due to advances in reproduc- tive medicine, there is an increasing need to introduce a safe and effective medication and technique to reduce pain during this procedure.To the best of our knowledge, this is the first study using ELMA among Iranian infertile women.

\section{Objectives}

The objective of this study was to assess the efficacy of the topical anesthetic (lidocaine 2.5\%/prilocaine 2.5\%) combination cream to reduce pain during and after HSG at five predefined steps (speculum application, cervical instrumentation of the tenaculum and cannula, at the end of uterine filling, speculum and cannula withdrawal and 30 minutes after the procedure).

\section{Patients and Methods}

This was a randomized double-blind clinical trial conducted from January 2011 to March 2012 at Mahdieh Hospital in Tehran. The study population consisted of women who were referred to the department of radiology for HSG during infertility investigation. Patients were considered eligible if: 1) They were alert, orientated and co-

Copyright (C) 2014, Tehran University of Medical Sciences and Iranian Society of Radiology; Published by Kowsar. This is an open-access article distributed under the terms of the Creative Commons Attribution-NonCommercial 4.0 International License (http://creativecommons.org/licenses/by-nc/4.0/) which permits copy and redistribute the material just in noncommercial usages, provided the original work is properly cited. 
operative to response to the visual analogue scale (VAS). 2) They signed the informed consent to participate in the clinical trial before entering the study.

Patients were excluded if: 1) They had a history of hypersensitivity to local anesthetics or were strictly prohibited to use local anesthetics. 2) They had used oral analgesics before the procedure. 3) They had a history of chronic pelvic pain or dysmenorrhea. 4) They could not cooperate or if there was any problem during the procedure such as difficulty in instrument application or difficulty in uterine expansion when there was need for more contrast injection.

A statistical type I error (alpha) of 0.05 , power of $80 \%$, mean difference of 1.6 in VAS ( 4.9 for the placebo group vs. 3.3 for the EMLA group) and an equal standard deviation of 2.5 for both groups altogether yielded a sample size of 40 in each group. Eighty-four women were assessed for eligibility; four were excluded from the study, two for stenosis of the internal cervical os and two because they used oral analgesics before the procedure.

A 10-point VAS $(0=$ no pain and $10=$ severe pain $)$ was used to represent the level of perceived pain intensity by patients. All patients were asked to sign a written consent after they were informed clearly about the HSG procedure, study design and VAS and its application. VAS is a unidimensional pain scale, first developed by Woodforde and Merskey (10). It is one of the most commonly accepted measures for the assessment of pain intensity (11). Traditionally, VAS consisted of a 100-mm horizontal line and the participant was asked to mark the line at the point that represents pain intensity $(0=$ no pain and 10 = pain as bad as it could possibly be), the score determined by using a ruler and measuring the distance from 0 to the patient's mark, providing a range of scores from 0 to 10 , on a white loose leaf of paper. Paper and pencil administration of the scores is an impractical technique in busy clinical settings and in this study (like similar recent studies); we used a common approach of numerical rating VAS that simply required patients to state whole numbers from 0 to 10 . The protocol was approved by Shahid Beheshti Medical Ethics Department and the Review Committee for Reproductive Medicine.

Patients were randomized to EMLA or control groups by a computer generated random allocation. We used block randomization without stratifying on confounder variables. A statistician generated the allocation sequence. Forty patients were randomized to the EMLA-treated group, and 40 to the placebo-control group. Before completing a questionnaire, participants were randomly allocated by specifying numbers on questionnaires by a secretary. The people who generated the allocation scheme were not involved in ascertaining eligibility, performing the procedure, and assessing the outcome. Both the HSG operator and the patient were blinded to the randomization. A researcher and HSG operator blinded to the randomization recorded the pain score specified by the patient at each stage of the procedure.

\subsection{Cream Application and HSG}

In patients allocated to the EMLA treated group, 5 grams of EMLA cream (Astra Zeneca) was applied tothe cervix by an applicator. In the control group, a placebo cream identical in color, smell and texture was used. The cream was placed on the uterine cervix using a vaginal speculum and a vaginal applicator 15 minutes before the procedure. The patient was placed in the lithotomy position on the radiology table. The procedure was performed under strictly sterile conditions. After insertion of the speculum, the cervix was localized and cleaned with povidone-iodine solution. Before cannulation, the cervix was grasped with a single-toothed tenaculum forceps at 12 o'clock position.

After cannula placement and removal of the speculum, installation of water-soluble contrast material was started slowly (visipaque $320 \mathrm{mg} / \mathrm{mL}$, each time 2-3 mL) and fluoroscopic images were obtained. At the end of the procedure and withdrawal of the instruments, the patient received observation care for an hour before discharge. In both groups, pain perception related to the HSG procedure was scored by VAS at five predefined steps:

Step 1: Speculum application

Step 2: Cervical instrumentation of the tenaculum and cannula

Step3: At the end of uterine filling

Step 4: Speculum and cannula withdrawal

Step 5: Half an hour after the procedure

\subsection{Statistical Analysis}

The pain scores of the two groups were compared. In order to compare quantitative variables between the two groups, non-parametric Mann - Whitney U test was applied. SPSS15 software for Windows (SPSS Inc., Chicago, Il, USA) was used for statistical analysis and a P-value of 0.05 or less was considered statistically significant.

\section{Results}

Forty women were randomized to the EMLA-treated group, and 40 to the placebo-control group. There were no statistical differences in patients' age (mean age of study group was $30.2 \pm 6.7$ and in the control group it was $29.1 \pm 4.9$ ). Patients' self-reported VAS pain scores are presented in Table 1 . There was no significant difference in the mean pain score between EMLA and placebo groups in pain perception during the entire procedure and 30 minutes after procedure.

In both groups, the most painful steps were step 3 (at the end of uterine filling) and step 2 (cervical instrumentation of the tenaculum and cannula). The detail and scores are illustrated in Figure 1.

Step 3 (at the end of uterine filling with contrast media) was significantly more painful than step2 (cervical instrumentation) in both groups (study group, $\mathrm{P}=0.013$; 


\begin{tabular}{|c|c|c|}
\hline & The Mean Pain Score & PValue \\
\hline Speculum application & & 0.602 \\
\hline EMLA & $2.9 \pm 2.6$ & \\
\hline Placebo & $3.3 \pm 2.5$ & \\
\hline Cervical instrumentation of the tenaculum and cannula & & 0.321 \\
\hline EMLA & $5.1 \pm 3.1$ & \\
\hline Placebo & $5.6 \pm 2.3$ & \\
\hline At the end of uterine filling & & 0.716 \\
\hline EMLA & $6.6 \pm 2.8$ & \\
\hline Placebo & $6.3 \pm 2.7$ & \\
\hline Speculum and cannula withdrawal & & 0.466 \\
\hline EMLA & $2.6 \pm 2.2$ & \\
\hline Placebo & $3 \pm 2.9$ & \\
\hline 30 minutes after procedure & & 0.596 \\
\hline EMLA & $1.7 \pm 1.5$ & \\
\hline Placebo & $2 \pm 2.6$ & \\
\hline
\end{tabular}

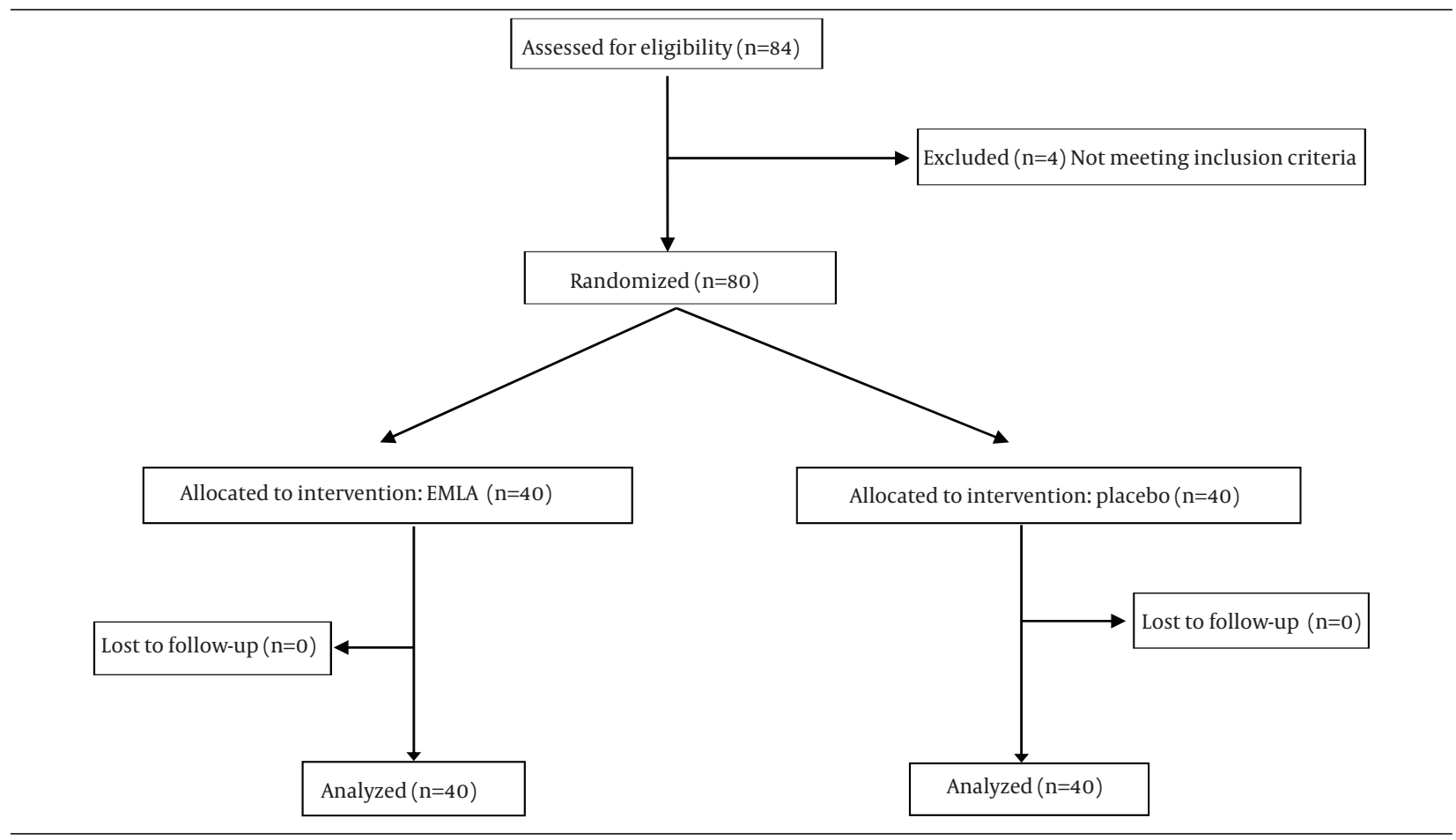

Figure 1. Flow diagram of analyzed data through phases of a parallel-randomized trial of two groups

control group, $\mathrm{P}<0.019)$. Step 3 was also significantly more painful than step 4 (speculum and cannula withdrawal, study group, $\mathrm{P}<0.001$; control group, $\mathrm{P}<0.001$ ). Cervical instrumentation (step 2) was significantly more painful than speculum application (step 1) in both groups (study group, $\mathrm{P}<0.001$; control group, $\mathrm{P}<0.001$ ). Step 5 (30 minutes after the procedure) was significantly less painful than step 4 (speculum and cannula withdrawal) in both groups (study group, $\mathrm{P}=0.022$; control group, $\mathrm{P}<$
0.047). Step 5 was also significantly less painful than step 1 (speculum application) (study group, $\mathrm{P}<0.003$; control group, $\mathrm{P}<0.005)$.

\section{Discussion}

HSG is a simple and cost effective investigation in assessing tubal patency. It is likely to have a key role in assessing tubal patency before undergoing infertility therapy, but the painful nature of HSG is the major reason the proce- 
dure has not gained wide acceptance between patients. The etiology of pain during the procedure is multifactorial and summarized as patient sensitivity to speculum application, cervical grasping, hydrostatic pressure of injection, stretched uterus, or peritoneal irritation (12).

In this study, different pain levels were recorded during the entire procedure. Pain started with speculum application and increased with cervical instrumentation and reached the peak at the end of uterine filling. This study shows substantial decrease with speculum and cannula withdrawal. Pain perception was reduced to the lowest point, 30 minutes after the procedure. This pain pattern was the same in EMLA and placebo groups.

In this study, there was no significant difference in efficacy between EMLA and placebo creams in pain perception through all steps of the procedure. There was no significant difference in long term pain perception half an hour after the HSG performance. Decreasing and increasing patterns of pain presentation during five predefined steps of HSG was the same in EMLA and placebo groups. There was no report of side effect and unexpected adverse events in the patients.

These results are not in agreement with previous studies. One randomized controlled study on 80 women compared the effectiveness of EMLA versus placebo and reported evidence of significant reduction in pain during cervical instrumentation that was found the most painful step of the procedure in their study (1). They applied topical ELMA on the cervix by cervical cap 30 minutes before HSG. Topical lidocaine spray $10 \%$ is also reported as an effective analgesic during the HSG procedure (13). The limitation of this study could be due to the subjective nature of pain and influencing pain factors including differences in individual physical susceptibility, psychological factors, and socio-cultural differences to whom VAS is applied. The effects of psychological and socio-cultural differences are minimized by using randomization to assign the participants to the two groups. Psychological influences of infertility on the perception of pain were comparable as well as socio-cultural differences between the two groups.
This study has not been designed to assess the association between pelvic pathology (pelvic adhesion and tubal occlusion) with more difficult and painful HSG performance. Further studies should consider the role of pelvic pathology in pain perception and also using intrauterine anesthetic combined with topical cervical anesthetic due to multifactorial pathogenesis of pain during HSG.

\section{References}

1. Liberty G, Gal M, Halevy-Shalem T, Michaelson-Cohen R, Galoyan $\mathrm{N}$, Hyman J, et al. Lidocaine-prilocaine (EMLA) cream as analgesia for hysterosalpingography: a prospective, randomized, controlled, double blinded study. Hum Reprod. 2007;22(5):1335-9.

2. Varpula M. Hysterosalpingography with a balloon cath eter versus a cannula: evaluation of patient pain. Radiology. 1989;172(3):745-7.

3. Tur-Kaspa I, Seidman DS, Soriano D, Greenberg I, Dor J, Bider D. Hysterosalpingography with a balloon catheter versus a metal cannula: a prospective, randomized, blinded comparative study. Hum Reprod.1998;13(1):75-7.

4. Moore DE. Pain associated with hysterosalpingography: Ethiodol versus Salpix media. Fertil Steril.1982;38(5):629-31.

5. Lindequist S, Justesen P, Larsen C, Rasmussen F. Diagnostic quality and complications of hysterosalpingography: oil- versus water-soluble contrast media--a randomized prospective study. Radiology. 1991;179(1):69-74.

6. Ahmad G, Duffy J, Watson AJ. Pain relief in hysterosalpingography. Cochrane Database Syst Rev. 2007(2):CD006106.

7. Cengiz M, Kafali H, Artuc $\mathrm{H}$, Baysal Z. Opioid analgesia for hysterosalpingography: controlled double-blind prospective trial with remifentanil and placebo. Gynecol Obstet Invest. 2006;62(3):168-72.

8. Frishman GN, Spencer PK, Weitzen S, Plosker S, Shafi F. The use of intrauterine lidocaine to minimize pain during hysterosalpingography: a randomized trial. Obstet Gynecol. 2004;103(6):1261-6.

9. Lorino CO, Prough SG, Aksel S, Abuzeid M, Alexander SE, Wiebe RH. Pain relief in hysterosalpingography. A comparison of analgesics. J Reprod Med.1990;35(5):533-6.

10. Woodforde JM, Merskey H. Some relationships between subjective measures of pain. J Psychosom Res. 1972;16(3):173-8.

11. Downie WW, Leatham PA, Rhind VM, Wright V, Branco JA, An derson JA. Studies with pain rating scales. Ann Rheum Dis. 1978;37(4):378-81.

12. Zhu YY, Mao YZ, Wu WL. Comparison of warm and cold contrast media for hysterosalpingography: a prospective, randomized study. Fertil Steril. 2012;97(6):1405-9.

13. Karasahin E, Alanbay I, Keskin U, Gezginc K, Baser I. Lidocaine 10\% spray reduces pain during hysterosalpingography: a randomized controlled trial. J Obstet Gynaecol Res. 2009;35(2):354-8. 